\title{
BL153 Partially Prevents High-Fat Diet Induced Liver Damage Probably via Inhibition of Lipid Accumulation, Inflammation, and Oxidative Stress
}

\author{
Jian Wang, ${ }^{1,2,3}$ Chi Zhang, ${ }^{2,4}$ Zhiguo Zhang, ${ }^{3,5}$ Qiang Chen,,5 Xuemian Lu, ${ }^{2,4}$ \\ Minglong Shao, ${ }^{2,4}$ Liangmiao Chen, ${ }^{2,4}$ Hong Yang, ${ }^{2,4}$ Fangfang Zhang, ${ }^{2,4}$ Peng Cheng, ${ }^{2,4}$ \\ Yi Tan, ${ }^{2,3}$ Ki-Soo Kim, ${ }^{6}$ Ki Ho Kim, ${ }^{7}$ Bochu Wang, ${ }^{1}$ and Young Heui Kim ${ }^{7}$ \\ ${ }^{1}$ College of Bioengineering, Chongqing University, Chongqing 400044, China \\ ${ }^{2}$ The Chinese-American Research Institute for Diabetic Complications, School of Pharmaceutical Sciences \& Key Laboratory of \\ Biotechnology Pharmaceutical Engineering, Wenzhou Medical University, Wenzhou 325035, China \\ ${ }^{3}$ Department of Pediatrics of the University of Louisville, The Kosair Children's Hospital Research Institute, Louisville, KY 20202, USA \\ ${ }^{4}$ Ruian Center of the Chinese-American Research Institute for Diabetic Complications, \\ The Third Affiliated Hospital of the Wenzhou Medical University, Wenzhou 325200, China \\ ${ }^{5}$ Department of Cardiology at the First Hospital \& School of Public Health, Jilin University, Changchun 130021, China \\ ${ }^{6}$ Bioland Biotec Co., Ltd., Zhangjiang Modern Medical Device Park, Pudong, Shanghai 201201, China \\ ${ }^{7}$ Bioland R\&D Center, 59 Songjeongni 2-gil, Byeongcheon, Dongnam, Cheonan, Chungnam 330-863, Republic of Korea
}

Correspondence should be addressed to Bochu Wang; wangbc2000@126.com and Young Heui Kim; yhkim1st@biolandkorea.com

Received 9 December 2013; Accepted 20 February 2014; Published 3 April 2014

Academic Editor: Si Jin

Copyright (c) 2014 Jian Wang et al. This is an open access article distributed under the Creative Commons Attribution License, which permits unrestricted use, distribution, and reproduction in any medium, provided the original work is properly cited.

\begin{abstract}
The present study was to investigate whether a magnolia extract, named BL153, can prevent obesity-induced liver damage and identify the possible protective mechanism. To this end, obese mice were induced by feeding with high fat diet (HFD, 60\% kcal as fat) and the age-matched control mice were fed with control diet (10\% kcal as fat) for 6 months. Simultaneously these mice were treated with or without BL153 daily at 3 dose levels $(2.5,5$, and $10 \mathrm{mg} / \mathrm{kg})$ by gavage. HFD feeding significantly increased the body weight and the liver weight. Administration of BL153 significantly reduced the liver weight but without effects on body weight. As a critical step of the development of NAFLD, hepatic fibrosis was induced in the mice fed with HFD, shown by upregulating the expression of connective tissue growth factor and transforming growth factor beta 1, which were significantly attenuated by BL153 in a dose-dependent manner. Mechanism study revealed that BL153 significantly suppressed HFD induced hepatic lipid accumulation and oxidative stress and slightly prevented liver inflammation. These results suggest that HFD induced fibrosis in the liver can be prevented partially by BL153, probably due to reduction of hepatic lipid accumulation, inflammation and oxidative stress.
\end{abstract}

\section{Introduction}

Obesity is becoming a health issue all over the world. It grows rapidly and always leads to severe complications such as cardiovascular disorder, diabetes, and cancer [1-3]. Liver is one of the most affected organs by obesity in the body, which leads to nonalcoholic fatty liver disease (NAFLD) [4]. NAFLD is a pathologic entity, including a spectrum of liver damage ranging from simple steatosis to nonalcoholic steatohepatitis (NASH), advanced fibrosis, and progression to cirrhosis [5]. The pathogenesis of NAFLD appears to involve a 2-hit process [6-8]. The first hit is the steatosis which is believed to be triggered by insulin resistance and the second hit which involves oxidative stress results in disease progression. The proinflammatory cytokines have been implicated in the pathogenesis of NAFLD and contribute to the increased risk for hepatocellular carcinoma. Therefore, attenuation of lipid accumulation, oxidative damage, and 
inflammation associated with obesity is expected to exert beneficial effects and thus be a potential novel therapeutic strategy for NAFLD.

Magnolia officinalis is regarded as Chinese traditional medicine and used in the clinical practice for a long time to treat various disorders $[9,10]$. Several constituents of the Magnolia such as honokiol, obovatol, and magnolol have been reported to have antioxidative $[11,12]$ and antiinflammatory effects [13-15]. Honokiol has been shown to have the effect of anti-inflammation by inhibiting NF- $\kappa \mathrm{B}$ activation, activin phosphorylation, and subsequent $\mathrm{I} \kappa \mathrm{B} \alpha$ degradation $[16,17]$. Consistent with the suppression effect of honokiol on NF- $\kappa \mathrm{B}$ is that honokiol decreases levels of NF$\kappa \mathrm{B}$ target genes including tumor necrosis factor (TNF-) $\alpha$, intercellular adhesion molecule (ICAM-) 1, and plasminogen activator inhibitor (PAI-) 1. Honokiol also plays critical role in scavenging reactive oxygen species via inhibition of TNF$\alpha$ mediated NADPH oxidase (NOX) pathway in hepatocytes [18]. In addition to honokiol, other two main constituents of Magnolia, magnolol, and obovatol also showed the antioxidative effect by attenuation of ROS generation and the subsequent reduction of NF- $\kappa \mathrm{B}$ activation $[19,20]$. Both oxidative stress and inflammation cause fibrosis, which can be prevented by attenuation of transforming growth factor beta 1 (TGF- $\beta 1$ ) and its downstream profibrotic factors such as connective tissue growth factor (CTGF) through inhibition of Smad-2/3 signaling pathway [21]. Furthermore, it was reported that magnolol regulated lipid metabolism by increase of fatty acid $\beta$-oxidation and lipolysis, finally reducing lipid accumulation in the tissue $[22,23]$.

The present study was to clarify whether BL153 has protective effects on HFD-induced liver damage and if so what is the possible mechanism. We found administration of BL153 significantly prevented chronic obesity-induced liver damage with the mechanism of reducing lipid accumulation and inhibiting inflammation and the associated oxidative stress.

\section{Material and Methods}

2.1. Experimental Protocols and Animals. Magnolia extract (BL153) was prepared as our previous report [24]. The major constituents and their structure of magnolia extract have been defined in previous studies [24-26]. All experiments involving mice conformed to the National Institutes of Health Guide for the Care and Use of Laboratory Animals and were approved by the University of Louisville Institutional Animal Care and Use Committee. Male C57BL/6J mice at 8 weeks of age were purchased from the Jackson Laboratory and housed in the University of Louisville Research Resources Center at $22^{\circ} \mathrm{C}$ with a 12 -hour light/dark cycle. Mice were randomly divided into five groups $(n=5)$ and fed either a control diet (Ctrl, 10\% kcal as fat; D12450B, Research Diets Inc. NJ) or a high fat diet (HFD, 60\% kcal as fat; D12492B, Research Diets Inc. NJ) with or without BL153 for six months: (1) Ctrl group: mice were fed a control diet and supplemented with $0.5 \%$ ethanol; (2) HFD group: mice were fed a HFD and supplemented with $0.5 \%$ ethanol; (3) HFD+2.5 mg/kg group: mice were fed a HFD and supplemented with BL153 at the dose of $2.5 \mathrm{mg} / \mathrm{kg}$; (4) HFD+5 mg/kg group: mice were fed a HFD and supplemented with BL153 at the dose of $5 \mathrm{mg} / \mathrm{kg}$; and (5) HFD+10 $\mathrm{mg} / \mathrm{kg}$ group: mice were fed a HFD and supplemented with BL153 at the dose of $10 \mathrm{mg} / \mathrm{kg}$. Selection of $5 \mathrm{mg} / \mathrm{kg}$ and $10 \mathrm{mg} / \mathrm{kg}$ for the present study was based on a previous study [25], where treatment with BL153 at these two dose levels for a week showed a significantly protective effect. Since the treatment in the present study is longer than that, we also included one lower dose of BL153 at $2.5 \mathrm{mg} / \mathrm{kg}$.

For preparing BL153 gavage solution, different doses of BL153 were dissolved into $100 \%$ ethanol and then diluted with $\mathrm{ddH}_{2} \mathrm{O}$ into final concentration of $1.0 \mathrm{mg} / \mathrm{mL}$ (high dose group), $0.5 \mathrm{mg} / \mathrm{mL}$ (middle dose group), and $0.25 \mathrm{mg} / \mathrm{mL}$ (low dose group) with final concentration of ethanol at $0.5 \%$, respectively. Therefore, the gavage volume was $1 \%(\mathrm{~mL} / \mathrm{g})$ of mouse body weight (e.g., 25 g mouse should be given $250 \mu \mathrm{L}$ ). Control groups were given same volume of $\mathrm{ddH}_{2} \mathrm{O}$ with $0.5 \%$ ethanol. During the six-month feeding, body weight was measured every month, and the gavage volume was justified based on the body weight change. At the end of experiment, all mice were sacrificed and livers were collected for further analysis.

\subsection{Histological Examination and Immunohistochemical} Staining. The fixed liver tissue was cut into $3 \mathrm{~mm}$ thickness blocks. The tissue blocks were embedded in paraffin and cut into $4 \mu \mathrm{m}$ slices. After being deparaffinized using xylene and ethanol dilutions and rehydration, the sections were stained with hematoxylin and eosin ( $\mathrm{H} \& \mathrm{E}, \mathrm{DAKO}$, Carpinteria, CA) to examine the tissue structure, inflammatory cell infiltration, necrosis, and lipid accumulation as described previously $[27,28]$. For immunohistochemical staining, sections were blocked with Superblock buffer (Pierce, Rockford, IL) for $30 \mathrm{~min}$. Sections were then incubated with proper primary antibodies in 1:200 dilutions overnight at $4^{\circ} \mathrm{C}$. After three washes with phosphate-buffered saline (PBS), these sections were incubated with horseradish peroxidase-labeled secondary antibody (Santa Cruz Biotechnology, Santa Cruz, CA) at room temperature for $1 \mathrm{~h}$, followed by color development with diaminobenzidine for $2 \mathrm{~min}$.

\subsection{Oil Red O Staining and Triglyceride Assay for Lipid} Accumulation. Cryosections from OCT-embedded tissue samples of the liver (10 mm thickness) were fixed in $10 \%$ buffered formalin for $5 \mathrm{~min}$ at room temperature, stained with Oil Red $\mathrm{O}$ for $1 \mathrm{~h}$, washed with $10 \%$ isopropanol, and then counterstained with hematoxylin for $30 \mathrm{~s}$. A Nikon microscope (Nikon, Melville, NY) was used to capture the Oil Red O-stained tissue sections at 40x magnification. For hepatic triglyceride levels assay, $200 \mathrm{mg}$ of hepatic tissues was homogenized at $4^{\circ} \mathrm{C}$ in $2.0 \mathrm{~mL}$ diluted Standard Diluent using a Polytron tissue homogenizer. After centrifugation at $10000 \times \mathrm{g}$ for $10 \mathrm{~min}$ at $4^{\circ} \mathrm{C}$, samples were diluted by the ratio of 1:5 using the diluted Standard Diluent. Then, the triglyceride levels in liver tissue were measured according to the manufacturers' instructions provided in the triglyceride colorimetric assay kit (Cayman Chemical, CA). 
2.4. Western Blot. Western blot assays were performed as described before [29]. Briefly, liver tissues were homogenized in RIPA lysis buffer (Santa Cruz Biotechnology, Santa Cruz, CA). Proteins were collected by centrifuging at $12,000 \mathrm{~g}$ at $4^{\circ} \mathrm{C}$. The sample of total protein was separated on 10\% SDS-PAGE gels and transferred to nitrocellulose membranes (Bio-Rad, Hercules, CA). These membranes were rinsed briefly in tris-buffered saline containing $0.05 \%$ Tween 20 (TBST), blocked in blocking buffer (5\% milk and $0.5 \% \mathrm{BSA}$ ) for $1 \mathrm{~h}$, and then incubated with different primary antibodies overnight at $4^{\circ} \mathrm{C}$, followed by three washes with TBST and incubation with secondary horseradish peroxidase-conjugated antibody for $1 \mathrm{~h}$ at room temperature. Antigen-antibody complexes were then visualized using ECL kit (Amersham, Piscataway, NJ). The primary antibodies used here include those against 3nitrotyrosine (3-NT, 1:2000, Millipore, Billerica, MA), 4hydroxynonenal (4-HNE, 1:2000, Alpha Diagnostic International, San Antonio, TX), ICAM-1 (1:500), CTGF and $\beta$-actin (1:1000, Santa Cruz Biotechnology, Santa Cruz, CA), PAI-1 (1:2000, BD Biosciences, Sparks, MD), TNFa (1:500), and TGF- $\beta 1$ (1:1000; Cell Signaling, Danvers, MA).

2.5. Statistical Analysis. Data were collected from five animals for each group and presented as mean \pm SD. Oneway ANOVA was used to determine general difference, followed by a post hoc Tukey's test for the difference between groups using Origin 7.5 laboratory data analysis and graphing software. Statistical significance was considered as $P<0.05$.

\section{Results}

3.1. HFD-Induced Obesity and the Effects of BL153. In order to identify whether BL153 can prevent obesity and the subsequent hepatic injury, HFD treatment was applied in this study to induce obesity mouse model. After 6 months of HFD feeding, the body weight was significantly increased, which indicated the establishment of the obesity mouse model (Figure 1(a)) but treatment with BL153 had no significant effects on HFD-induced body weight gain (Figure 1(a)). Additionally, HFD also significantly increased liver weight (Figure 1(b)) and the ratio of liver weight to tibia length (Figure 1(c)); treatment with BL153 slightly prevented HFDinduced liver weight increase (Figure 1(b)) but significantly prevented the ratio of liver weight to tibia length (Figure 1(c)), a more reasonable indicator of liver hypotrophy.

3.2. BL153 Attenuated HFD-Induced Hepatic Fibrosis. Liver weight increase is a feature of hepatic hypertrophy which is closely associated with liver fibrosis [30-32]. Moreover, fibrosis is a key step of the development of NAFLD [33, 34]. We next identified whether administration of BL153 prevents hepatic fibrosis under obese conditions. Western blot assay and immunohistochemical staining revealed that HFD treatment significantly upregulated hepatic CTGF expression which was significantly attenuated by administration of BL153 in a dose-dependent manner (Figures 2(a) and 2(b)). In order to further confirm our findings about the antifibrotic effect of BL153 in HFD fed mice, we also examined the expression of another classical fibrotic marker TGF- $\beta 1$. Similar protective effects were observed that HFD significantly increased hepatic TGF- $\beta 1$ expression, which was remarkably reduced by treatment of BL153 in a dose-dependent manner (Figure 2(c)).

3.3. Effects of BL153 on HFD-Induced Hepatic Steatosis. The above study revealed that BL153 significantly prevented HFDinduced hepatic hypertrophy and fibrosis. And lipid accumulation is the first step of NAFLD development $[35,36]$. Thus, we tried to determine whether BL153 can prevent HFDinduced hepatic steatosis. Liver pathological examination with H\&E staining is presented in Figure 3(a). The hepatic cell structure in Ctrl group was normal. However, HFD feeding increased hepatic damage with obviously hepatic necrosis (Figure 3(a)). Further examination of hepatic lipid accumulation status with Oil red $\mathrm{O}$ staining and triglyceride level assay revealed that HFD feeding significantly induced hepatic lipid accumulation compared to Ctrl group (Figures 3(b) and 3(c)). Administration of BL153 significantly, but not completely, prevented HFD-induced hepatic lipid accumulation (Figures $3(b)$ and 3(c)).

3.4. BL153 Attenuated HFD-Induced Hepatic Inflammation. Inflammation is the main pathological consequence of HFDinduced obesity characterized by release of inflammatory factors which contributes to insulin resistance [37-39]. Thus, we determined whether BL153 can prevent HFD-induced hepatic inflammation. The protein expression of classic inflammatory factors including ICAM-1, TNF- $\alpha$, and PAI- 1 was detected. Western blot assay revealed that HFD significantly upregulated the expression of TNF- $\alpha$ (Figure 4(a)), ICAM-1 (Figure 4(b)), and PAI-1 (Figure 4(c)) in the liver. However, all three doses of BL153 treatment significantly attenuated HFD-induced upregulation of TNF- $\alpha$ (Figure 4(a)), ICAM1 (Figure 4(b)), and PAI-1 (Figure 4(c)), while no significant differences among the three doses of BL153 treatment were observed.

3.5. BL153 Attenuated HFD-Induced Hepatic Oxidative Stress. HFD-induced obesity generally leads to oxidative stress via release of multiple adipokines which in turn generates excessive reactive oxygen species [40]. Furthermore, obesityassociated inflammation is an oxidative stress enhancer, which interacts with each other and causes a vicious circle, promoting the development of insulin resistance $[41,42]$. Thus, we next determined whether BL153 can prevent HFD-induced oxidative stress measured by 3 -NT as an index of nitrosative damage (Figure 5(a)) and 4HNE (lipid peroxide) as an index of oxidative damage (Figure 5(b)). The result showed that HFD feeding significantly upregulated the expression of 3-NT and 4-HNE in the liver, which were significantly attenuated by BL153 treatment in a dose-dependent manner (Figures 5(a) and $5(b))$. 


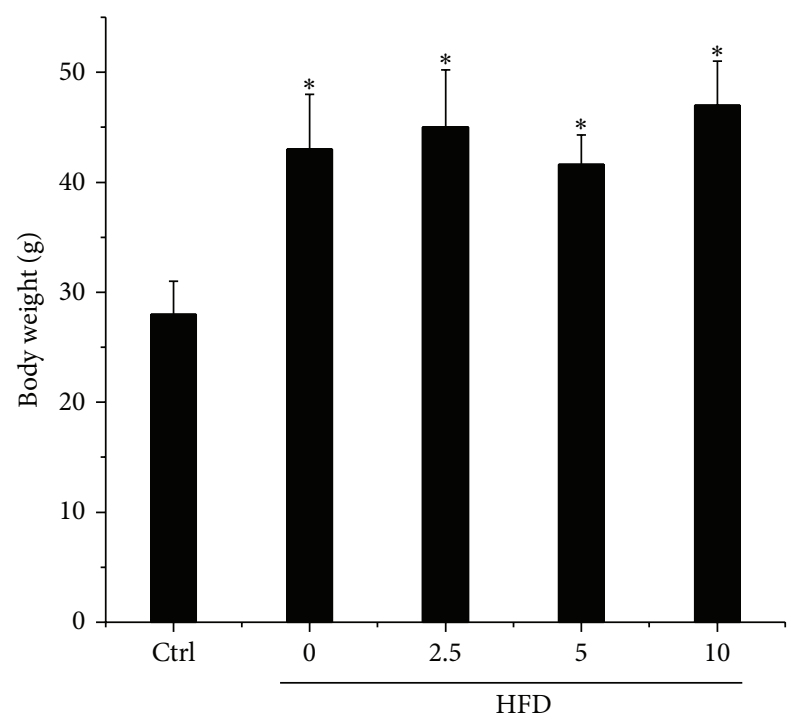

(a)

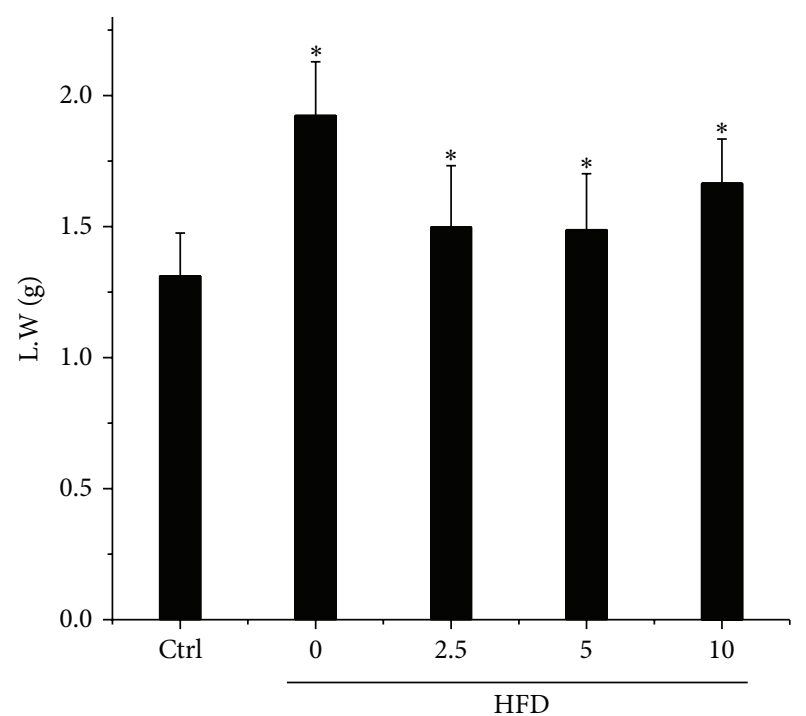

(b)

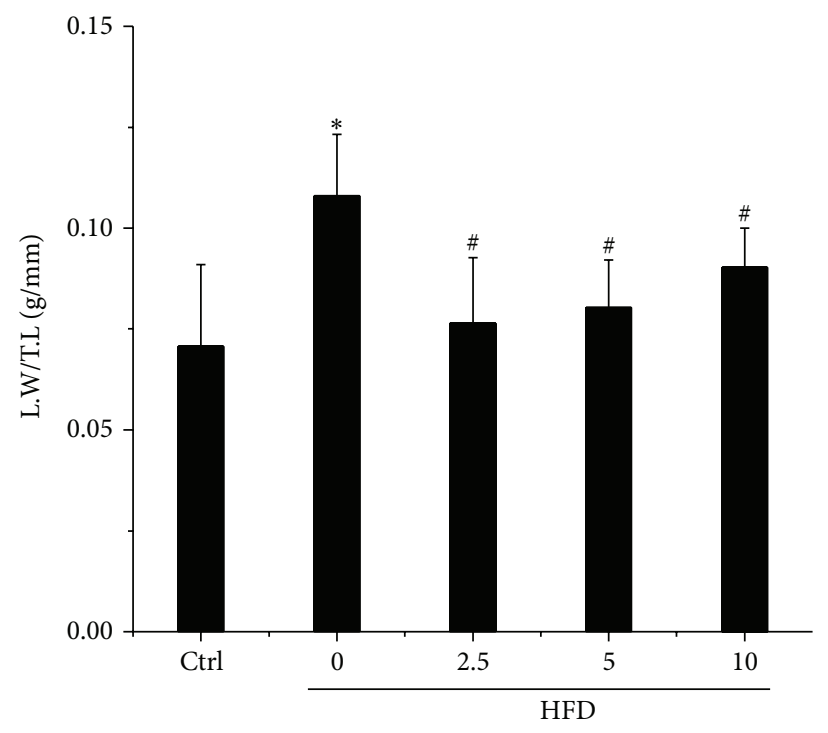

(c)

FIGURE 1: Effects of BL153 on body weight, liver weight, and the ratio of liver weight to tibia length. Mice were fed HFD to induce obesity; mouse models were simultaneously treated with or without BL153 at three dose levels $(2.5,5 \mathrm{or} 10 \mathrm{mg} / \mathrm{kg}$ body weight) by gavage. The body weight (a) was monitored at 6 months after HFD feeding. Then, the mice were sacrificed and the liver weight (b) and the ratio of liver weight to tibia length (c) were examined. Data were presented as means \pm SD $\left(n=5\right.$ at least in each group). ${ }^{*} P<0.05$ versus Ctrl group. ${ }^{\#} P<0.05$ versus HFD group. L.W = liver weight; T.L = tibia length; Ctrl: control; HFD: high fat diet.

\section{Discussion}

Obesity is currently a worldwide epidemic and among the most challenging health conditions. A major metabolic consequence of obesity is insulin resistance which underlies the pathogenesis of the metabolic syndrome such as NAFLD, the hepatic manifestation of obesity and metabolic syndrome $[6,43]$. NAFLD is considered to be the most common liver disorder in western countries, estimated to affect at least one-quarter of the general population and rising up to $90 \%$ in morbidly obese individuals $[44,45]$. It comprises a disease spectrum ranging from steatosis (fatty liver), through NASH, to fibrosis and ultimately liver cirrhosis. Previous studies mentioned that the pathogenesis of NAFLD commonly can be divided into two hits. The first hit, hepatic triglyceride accumulation (steatosis), increases susceptibility of the liver to injury mediated by second hit, such as inflammation and oxidative stress, which in turn lead to fibrosis $[8,46]$. Therefore, finding a proper way which can simultaneously target both of the first and second hits, that is, lipid accumulation, oxidative stress, inflammation, and fibrosis, might be a potential approach to prevent the development of NAFLD in clinics. 

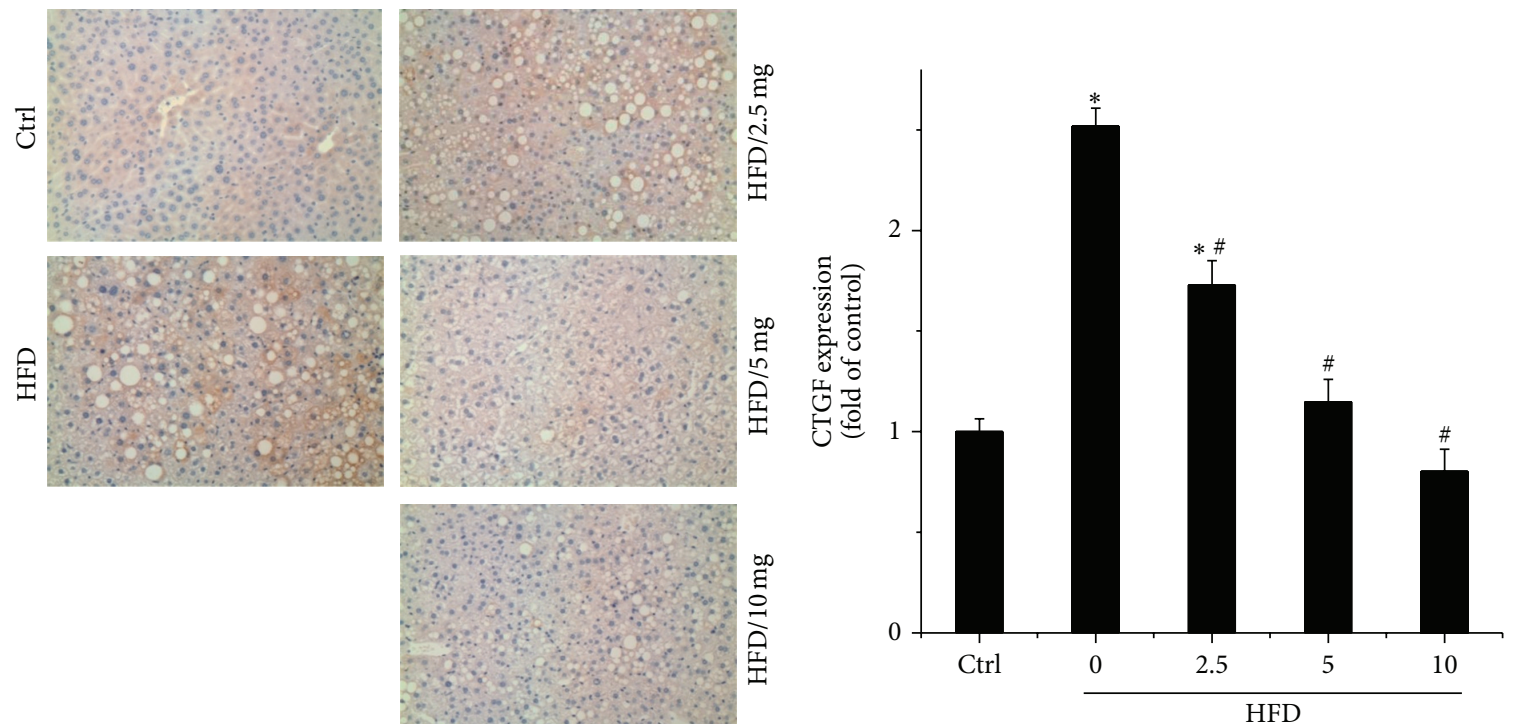

(a)
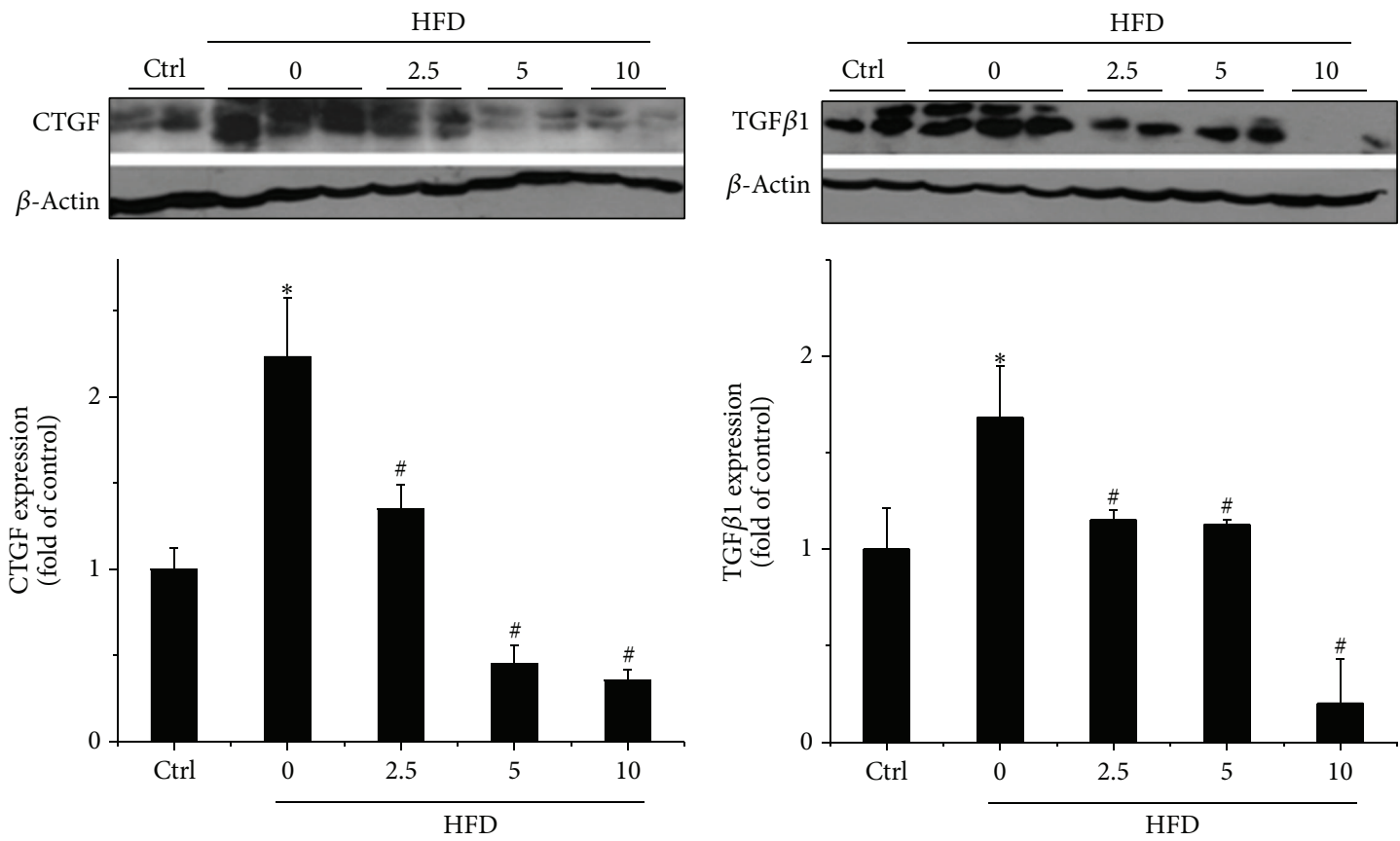

(b)

(c)

Figure 2: Effects of BL153 on HFD-induced fibrosis in the liver. The expression of fibrotic molecular maker CTGF was detected by immunohistochemical staining (a), and both CTGF (b) and TGF- $\beta 1$ (c) were also detected by Western blot. Data were presented as means \pm $\mathrm{SD}$ ( $n=5$ at least in each group). ${ }^{*} P<0.05$ versus Ctrl group. ${ }^{\#} P<0.05$ versus HFD group. CTGF: connective tissue growth factor; TGF- $\beta 1$ : transforming growth factor $\beta 1$; Ctrl: control; HFD: high fat diet.

In the present study, we provide the first evidence that magnolia extract, BL153, attenuated obesity-associated liver damage in a HFD-induced obesity mouse model. Most importantly, BL153 treatment significantly attenuated obesity caused liver pathological changes, including hepatic hypertrophy, lipid accumulation, fibrosis, inflammation, and oxidative stress.

Magnolia has been used as Chinese traditional medicine to treat various disorders $[9,10]$. It is reported that magnolol, a main compound isolated from Magnolia bark, reduces the number of intracellular stored lipid droplets by enhancing lipolysis and thus inhibits the formation of intracellular cholesterol esters [47]. It is unclear whether the liver weight lowering and anti-inflammation effects of BL153 are attributed to scavenging of lipid accumulation in liver. In our study, we examined the hepatic lipid accumulation in both HFD-fed and standard diet-fed mice by Oil Red $\mathrm{O}$ staining and triglyceride assay (Figure 3 ). The result showed that the 

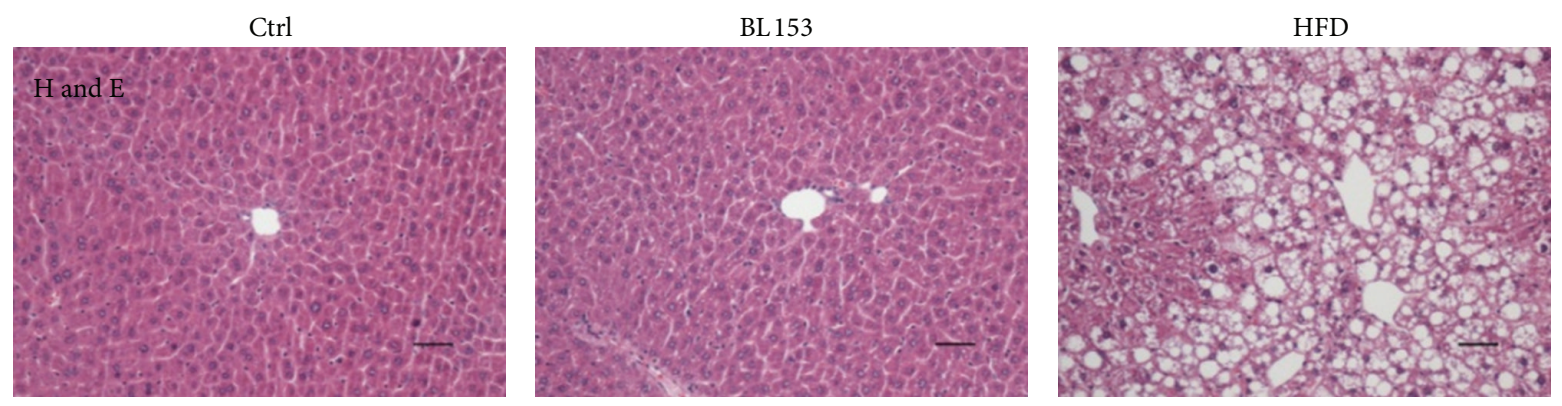

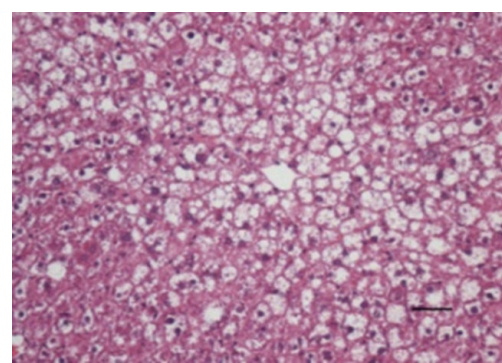

$\mathrm{HFD}+2.5 \mathrm{mg}$

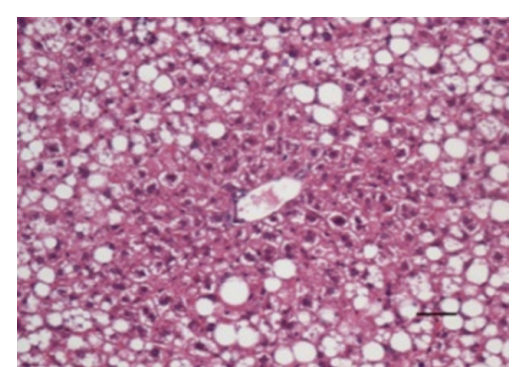

$\mathrm{HFD}+5.0 \mathrm{mg}$

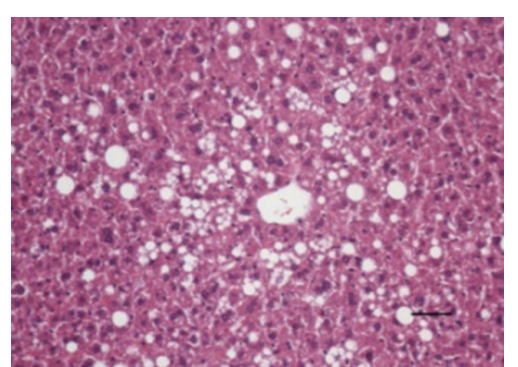

$\mathrm{HFD}+10.0 \mathrm{mg}$

(a)

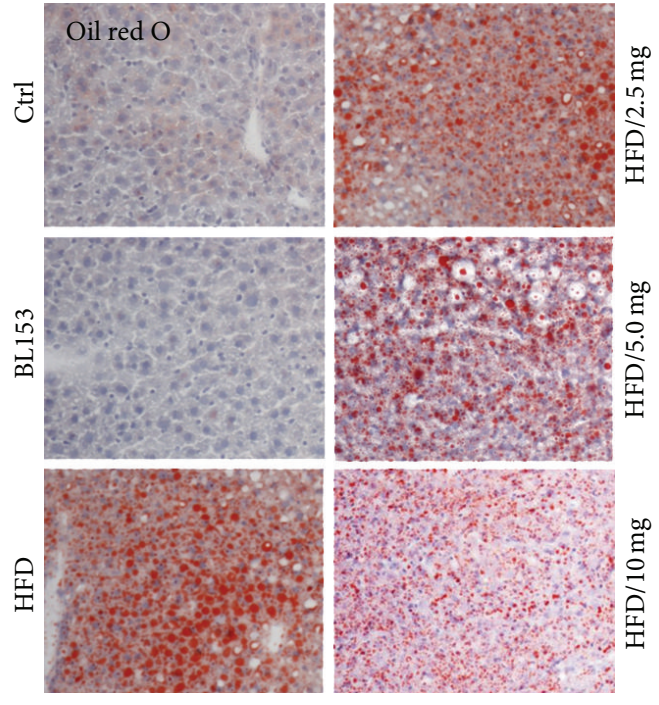

(b)

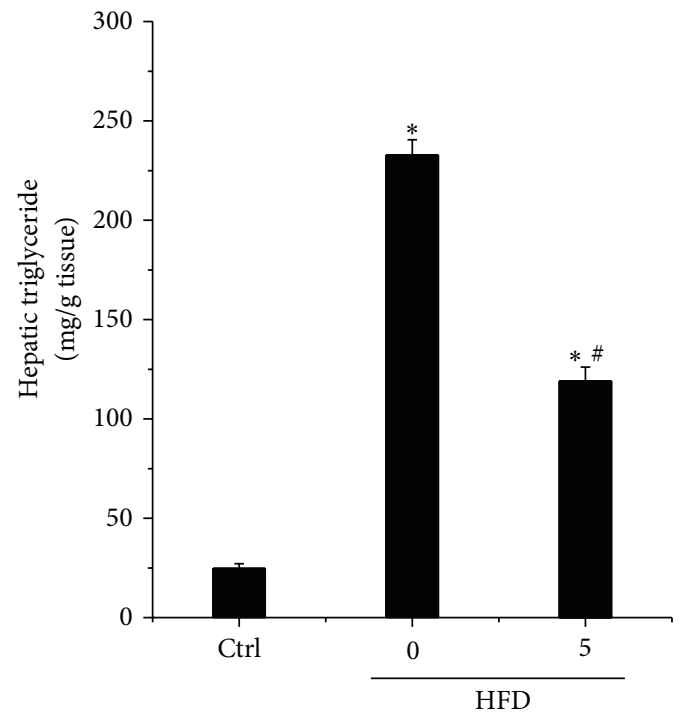

(c)

FIGURE 3: Effects of BL153 on HFD-induced hepatic lipid accumulation. Hepatic morphological changes were examined microscopically with H\&E staining ( (a) original magnification $=40)$. Hepatic lipid accumulation was examined by Oil Red O staining $((\mathrm{b})$ original magnification $=40)$ and triglyceride level assay (c). Data were presented as means $\pm \mathrm{SD}\left(n=5\right.$ at least in each group). ${ }^{*} P<0.05$ versus Ctrl group; ${ }^{*} P<0.05$ versus HFD group. Ctrl: control; HFD: high fat diet.

fat droplets were obviously observed in the liver of HFDfed mice associated with hepatic necrosis, which were significantly inhibited by BL153 treatment (Figure 3). The current study revealed that lowering hepatic lipid accumulation was the key mechanism of BL153 to fight against the first hit of NAFLD.

Liver weight increase is a main feature of hepatic hypertrophy associated with hepatic fibrosis, which is a key step during the development of NAFLD [48, 49]. Growing evidence demonstrated that Magnolia also showed great beneficial effect on antifibrosis. Administration of Magnolia cannot only prevent the cardiac fibrosis induced by ischemia/reperfusion but also the renal fibrosis induced by TGF- $\beta 1[21,50]$. In the present study, we further confirmed that HFD significantly upregulated hepatic CTGF and TGF$\beta 1$ expression, which were remarkably attenuated by BL153 treatment in a dose-dependent manner (Figure 2).

As we know, inflammation and the associated oxidative stress also participate in the development of NAFLD. Moreover, other studies also mentioned that another constituent 

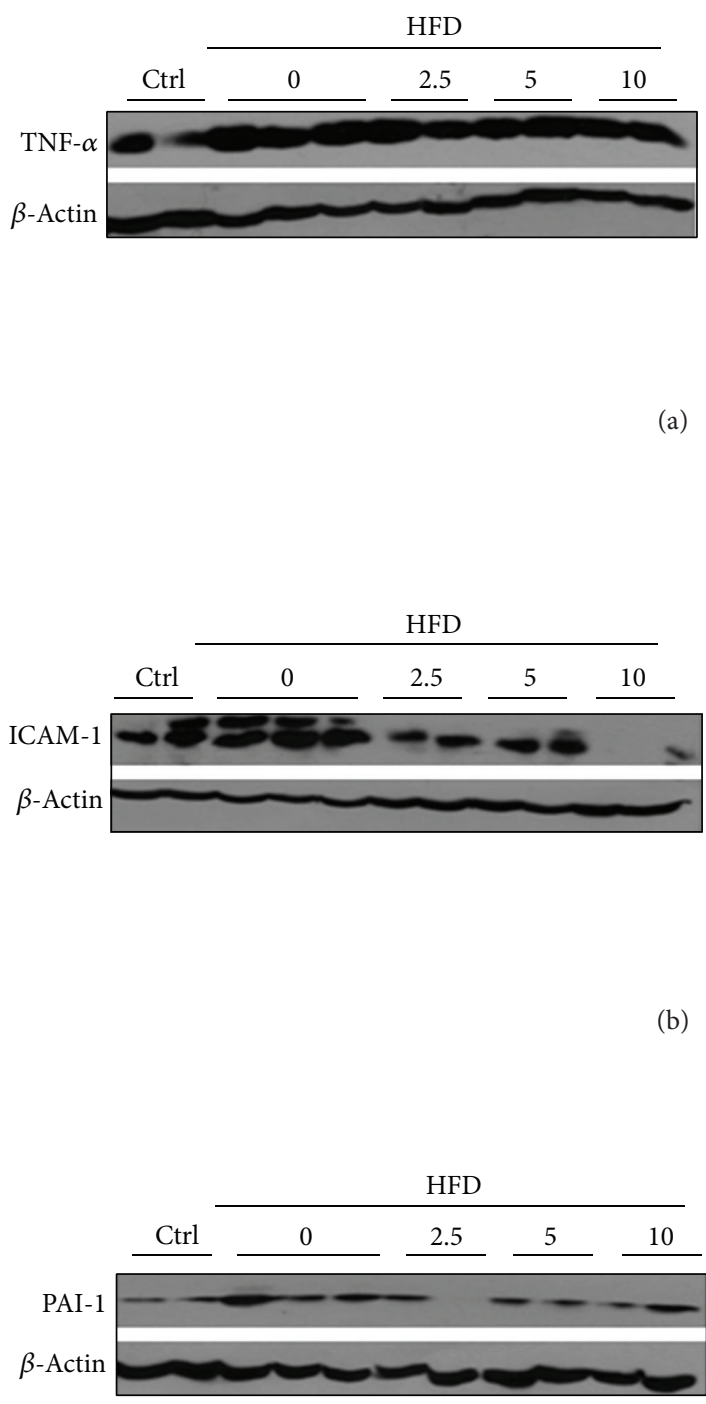

(a)

(b)
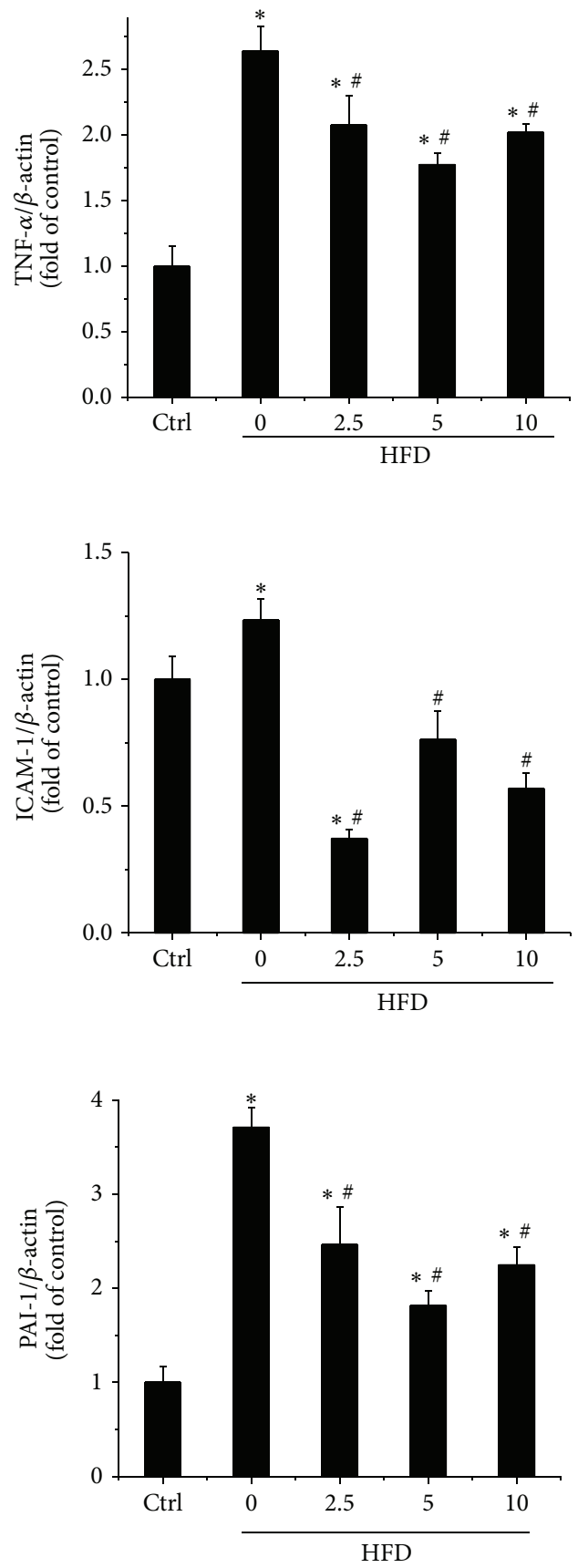

(c)

FIGURE 4: Effects of BL153 on HFD-induced hepatic inflammation. The expression of inflammatory factors, including TNF-a (a), ICAM-1 (b), and PAI-1 (c) was examined by Western blot. Data were presented as mean $\pm \operatorname{SD}\left(n=5\right.$ at least in each group). ${ }^{*} P<0.05$ versus Ctrl group; ${ }^{\#} P<0.05$ versus HFD group. TNF-a: tumor necrosis factor a; ICAM-1: intercellular adhesion molecule-1; PAI-1: plasminogen activator inhibitor-1; Ctrl: control; HFD: high fat diet.

of Magnolia, honokiol, plays inhibiting role on lipid accumulation-induced inflammation and oxidative stress [1315, 51]. Therefore, we tried to determine whether antiinflammation and antioxidation are the missing mechanisms of BL153 on preventing pathological process of HFD-induced liver damage. Our results indicated that HFD significantly upregulated the expression of hepatic inflammatory factors including TNF- $\alpha$, ICAM-1, and PAI-1 (Figure 4) as well as the marker of oxidative stress including 3-NT and 4-HNE (Figure 5). Administration of BL153 significantly attenuated oxidative stress shown by decrease of 3-NT and 4-HNE expressions in the liver with a dose-dependent manner (Figure 5). BL153 also remarkably inhibited hepatic inflammatory factor expressions without significant differences 

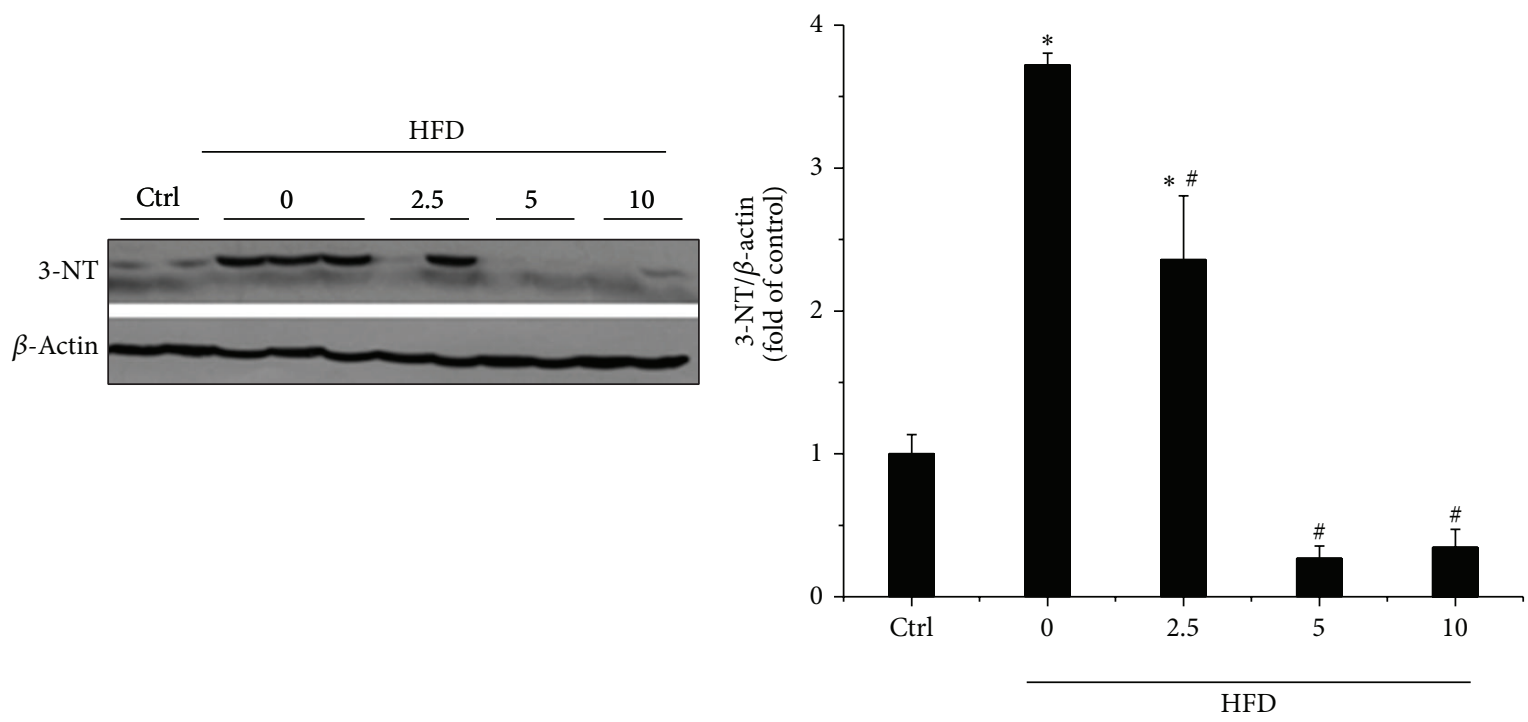

(a)
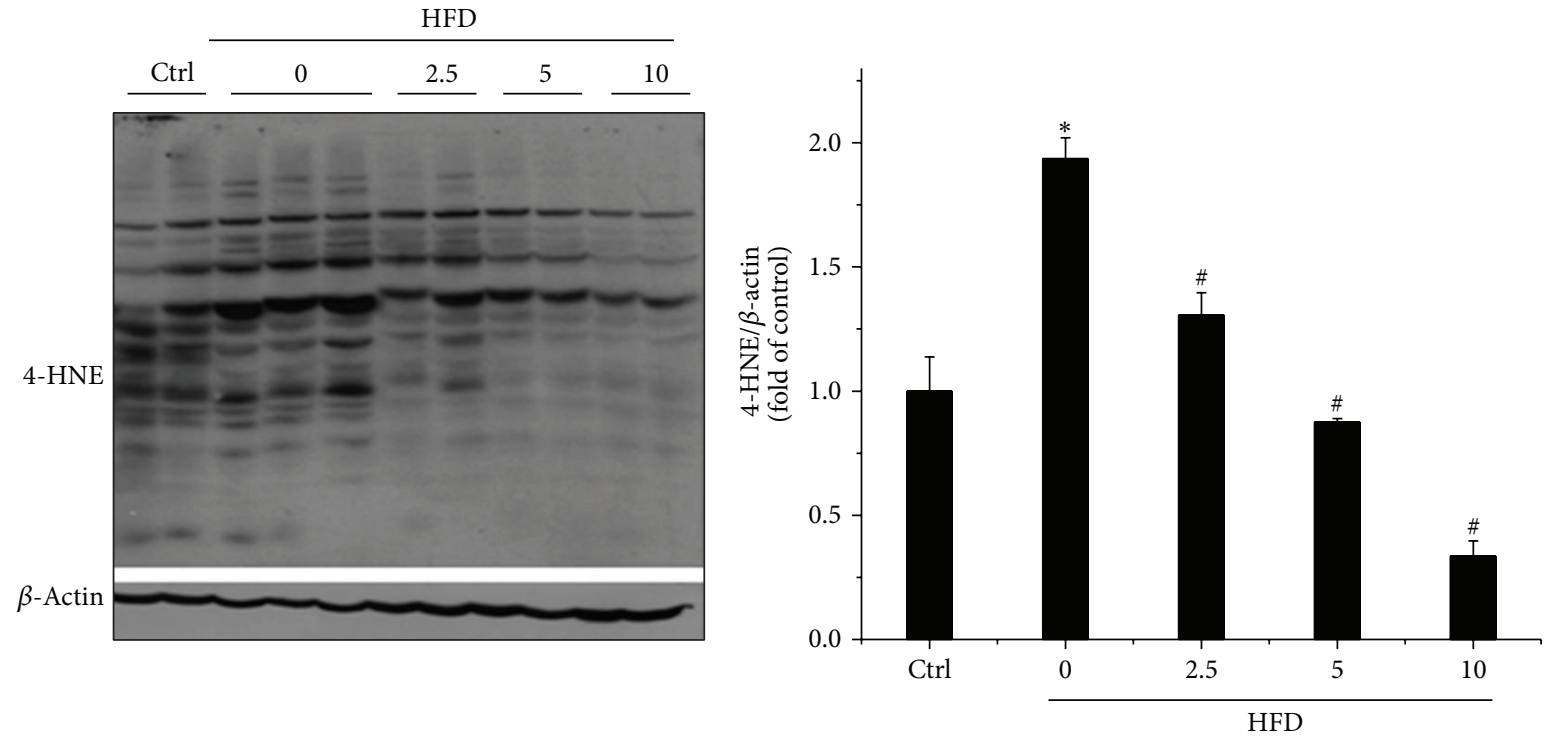

(b)

FIGURE 5: Effects of BL153 on HFD-induced hepatic oxidative stress. Hepatic expression of oxidative stress marker 3-NT (a) and 4-HNE (b) was examined by Western blot. Data were presented as mean $\pm \mathrm{SD}\left(n=5\right.$ at least in each group). ${ }^{*} P<0.05$ versus Ctrl group; ${ }^{\#} P<0.05$ versus 3-NT: 3-nitrotyrosine; 4-HNE: 4-hydroxynonenal HFD group. Ctrl: control; HFD: high fat diet.

among three dose level treatments (Figure 4), which implied that BL153 had different sensitivity to HFD-induced inflammation and oxidative stress.

In summary, the fat liver damage is a main consequence of the development of obesity induced by HFD. The magnolia extract BL153 can simultaneously induce beneficial effects on HFD-induced liver damage by inhibiting hepatic lipid accumulation, inflammation, oxidative stress, hypertrophy, and fibrosis.

\section{Conflict of Interests}

The authors have no conflict of interests to be declared.

\section{Authors' Contribution}

Jian Wang and Chi Zhang equally contributed to this paper.

\section{Acknowledgments}

This study was supported, in part, by Chungbuk Technopark Grant Biointernational Collaborating Research, funded by Chungbuk Province, Republic of Korea, and a collaborative project between University of Louisville and BioLand Co. Ltd. for "Screening Antidiabetes and/or Obesity Naturally Extracted Compounds," funded by BioLand Co., Ltd. (100826 Bioland). This study was also supported by the National 
Science Foundation of China (81000294 \& 81370917 to CZ) and the Research Development Fund of Wenzhou Medical University (QTJ13005 to CZ and QTJ 13007 to YT). The authors thank Dr. Lu Cai, who is from the Kosair Children's Hospital Research Institute of the University of Louisville, for his proof reading of this revised paper.

\section{References}

[1] P. Poirier, "Targeting abdominal obesity in cardiology: can we be effective?" The Canadian Journal of Cardiology, vol. 24, supplement D, pp. 13D-17D, 2008.

[2] C. L. Ogden, S. Z. Yanovski, M. D. Carroll, and K. M. Flegal, "The epidemiology of obesity," Gastroenterology, vol. 132, no. 6, pp. 2087-2102, 2007.

[3] Z. Lin, Z. Zhou, Y. Liu et al., "Circulating FGF21 levels are progressively increased from the early to end stages of chronic kidney diseases and are associated with renal function in Chinese," PLoS ONE, vol. 6, no. 4, Article ID e18398, 2011.

[4] G. C. Farrell and C. Z. Larter, "Nonalcoholic fatty liver disease: from steatosis to cirrhosis," Hepatology, vol. 43, supplement 1 , no. 2, pp. S99-S112, 2006.

[5] L. A. Adams, J. F. Lymp, J. St. Sauver et al., "The natural history of nonalcoholic fatty liver disease: a population-based cohort study," Gastroenterology, vol. 129, no. 1, pp. 113-121, 2005.

[6] J. K. Dowman, J. W. Tomlinson, and P. N. Newsome, "Pathogenesis of non-alcoholic fatty liver disease," QJM, vol. 103, no. 2, pp. 71-83, 2010.

[7] K. F. Tacer and D. Rozman, "Nonalcoholic fatty liver disease: focus on lipoprotein and lipid deregulation," Journal of Lipids, vol. 2011, Article ID 783976, 14 pages, 2011.

[8] C. P. Day and O. F. W. James, "Steatohepatitis: a tale of two "hits"?" Gastroenterology, vol. 114, no. 4, pp. 842-845, 1998.

[9] H. Kuribara, E. Kishi, N. Hattori, M. Okada, and Y. Maruyama, "The anxiolytic effect of two oriental herbal drugs in Japan attributed to honokiol from magnolia bark," Journal of Pharmacy and Pharmacology, vol. 52, no. 11, pp. 1425-1429, 2000.

[10] Y. Li, C. Xu, Q. Zhang, J. Y. Liu, and R. X. Tan, "In vitro antiHelicobacter pylori action of 30 Chinese herbal medicines used to treat ulcer diseases," Journal of Ethnopharmacology, vol. 98, no. 3, pp. 329-333, 2005.

[11] V. K. Bajpai, J. I. Yoon, and S. C. Kang, "Antioxidant and antidermatophytic activities of essential oil and extracts of Magnolia liliflora Desr," Food and Chemical Toxicology, vol. 47, no. 10, pp. 2606-2612, 2009.

[12] S. Dikalov, T. Losik, and J. L. Arbiser, "Honokiol is a potent scavenger of superoxide and peroxyl radicals," Biochemical Pharmacology, vol. 76, no. 5, pp. 589-596, 2008.

[13] J. Ock, H. S. Han, S. H. Hong et al., "Obovatol attenuates microglia-mediated neuroinflammation by modulating redox regulation," British Journal of Pharmacology, vol. 159, no. 8, pp. 1646-1662, 2010.

[14] Y.-R. Lin, H.-H. Chen, C.-H. Ko, and M.-H. Chan, "Effects of honokiol and magnolol on acute and inflammatory pain models in mice," Life Sciences, vol. 81, no. 13, pp. 1071-1078, 2007.

[15] M. E. Munroe, J. L. Arbiser, and G. A. Bishop, "Honokiol, a natural plant product, inhibits inflammatory signals and alleviates inflammatory arthritis," The Journal of Immunology, vol. 179, no. 2, pp. 753-763, 2007.
[16] S. I. Grivennikov and M. Karin, "Dangerous liaisons: STAT3 and NF- $\kappa$ B collaboration and crosstalk in cancer," Cytokine and Growth Factor Reviews, vol. 21, no. 1, pp. 11-19, 2010.

[17] M. Karin, "NF- $\kappa$ B and cancer: mechanisms and targets," Molecular Carcinogenesis, vol. 45, no. 6, pp. 355-361, 2006.

[18] E.-J. Park, S.-Y. Kim, Y.-Z. Zhao, and D. H. Sohn, "Honokiol reduces oxidative stress, c-jun-NH2-terminal kinase phosphorylation and protects against glycochenodeoxycholic acidinduced apoptosis in primary cultured rat hepatocytes," Planta Medica, vol. 72, no. 7, pp. 661-664, 2006.

[19] M. S. Choi, S. H. Lee, H. S. Cho et al., "Inhibitory effect of obovatol on nitric oxide production and activation of NF- $\kappa \mathrm{B} / \mathrm{MAP}$ kinases in lipopolysaccharide-treated RAW 264.7cells," European Journal of Pharmacology, vol. 556, no. 1-3, pp. 181-189, 2007.

[20] H.-C. Ou, F.-P. Chou, W. H.-H. Sheu, S.-L. Hsu, and W.-J. Lee, "Protective effects of magnolol against oxidized LDL-induced apoptosis in endothelial cells," Archives of Toxicology, vol. 81, no. 6, pp. 421-432, 2007.

[21] C.-K. Chiang, M.-L. Sheu, Y.-W. Lin et al., "Honokiol ameliorates renal fibrosis by inhibiting extracellular matrix and proinflammatory factors in vivo and in vitro," British Journal of Pharmacology, vol. 163, no. 3, pp. 586-597, 2011.

[22] J.-H. Chiu, J.-C. Wang, W.-Y. Lui, C.-W. Wu, and C.-Y. Hong, "Effect of magnolol on in vitro mitochondrial lipid peroxidation and isolated cold-preserved warm-reperfused rat livers," Journal of Surgical Research, vol. 82, no. 1, pp. 11-16, 1999.

[23] M. H. Lin, H. T. Chao, and C. Y. Hong, "Magnolol protects human sperm motility against lipid peroxidation: a sperm head fixation method," Archives of Andrology, vol. 34, no. 3, pp. 151156,1995

[24] W. Cui, Y. Wang, Q. Chen et al., “Magnolia extract (BL153) ameliorates kidney damage in a high fat diet-induced obesity mouse model," Oxidative Medicine and Cellular Longevity, vol. 2013, Article ID 367040, 9 pages, 2013.

[25] Y. K. Lee, D. Y. Yuk, T. I. Kim et al., "Protective effect of the ethanol extract of Magnolia officinalis and 4-O-methylhonokiol on scopolamine-induced memory impairment and the inhibition of acetylcholinesterase activity," Journal of Natural Medicines, vol. 63, no. 3, pp. 274-282, 2009.

[26] Y.-J. Lee, Y. M. Lee, C.-K. Lee, J. K. Jung, S. B. Han, and J. T. Hong, "Therapeutic applications of compounds in the Magnolia family," Pharmacology and Therapeutics, vol. 130, no. 2, pp. 157176, 2011.

[27] Y. Song, C. Li, and L. Cai, "Fluvastatin prevents nephropathy likely through suppression of connective tissue growth factormediated extracellular matrix accumulation," Experimental and Molecular Pathology, vol. 76, no. 1, pp. 66-75, 2004.

[28] G. Zhou, C. Li, and L. Cai, "Advanced glycation end-products induce connective tissue growth factor-mediated renal fibrosis predominantly through transforming growth factor $\beta$ independent pathway," The American Journal of Pathology, vol. 165, no. 6, pp. 2033-2043, 2004.

[29] C. Zhang, Y. Tan, W. Guo et al., "Attenuation of diabetesinduced renal dysfunction by multiple exposures to low-dose radiation is associated with the suppression of systemic and renal inflammation," The American Journal of PhysiologyEndocrinology and Metabolism, vol. 297, no. 6, pp. E1366-E1377, 2009.

[30] S. C. Faria, K. Ganesan, I. Mwangi et al., "MR imaging of liver fibrosis: current state of the art," Radiographics, vol. 29, no. 6, pp. 1615-1635, 2009. 
[31] D. C. Rockey, "Noninvasive assessment of liver fibrosis and portal hypertension with transient elastography," Gastroenterology, vol. 134, no. 1, pp. 8-14, 2008.

[32] C. C. Choong, S. K. Venkatesh, and E. P. Siew, "Accuracy of routine clinical ultrasound for staging of liver fibrosis," Journal of Clinical Imaging Science, vol. 2, article 58, 2012.

[33] V. Nobili, A. Alisi, A. Vania, C. Tiribelli, A. Pietrobattista, and G. Bedogni, "The pediatric NAFLD fibrosis index: a predictor of liver fibrosis in children with non-alcoholic fatty liver disease," BMC Medicine, vol. 7, article 21, 2009.

[34] D. A. Sass, P. Chang, and K. B. Chopra, "Nonalcoholic fatty liver disease: a clinical review," Digestive Diseases and Sciences, vol. 50, no. 1, pp. 171-180, 2005.

[35] Y. Kawano and D. E. Cohen, "Mechanisms of hepatic triglyceride accumulation in non-alcoholic fatty liver disease," Journal of Gastroenterology, vol. 48, no. 4, pp. 434-441, 2013.

[36] H.-R. Yao, J. Liu, D. Plumeri et al., "Lipotoxicity in HepG2 cells triggered by free fatty acids," The American Journal of Translational Research, vol. 3, no. 3, pp. 284-291, 2011.

[37] A. B. Goldfine, R. Silver, W. Aldhahi et al., "Use of salsalate to target inflammation in the treatment of insulin resistance and type 2 diabetes," Clinical and Translational Science, vol. 1, no. 1, pp. 36-43, 2008.

[38] S. E. Shoelson, L. Herrero, and A. Naaz, "Obesity, inflammation, and insulin resistance," Gastroenterology, vol. 132, no. 6, pp. 2169-2180, 2007.

[39] S. E. Shoelson, J. Lee, and A. B. Goldfine, "Inflammation and insulin resistance," Journal of Clinical Investigation, vol. 116, no. 7, pp. 1793-1801, 2006.

[40] A. Fernandez-Sanchez, E. Madrigal-Santillan, M. Bautista et al., "Inflammation, oxidative stress, and obesity," International Journal of Molecular Sciences, vol. 12, no. 5, pp. 3117-3132, 2011.

[41] H. Xu, G. T. Barnes, Q. Yang et al., "Chronic inflammation in fat plays a crucial role in the development of obesity-related insulin resistance," Journal of Clinical Investigation, vol. 112, no. 12, pp. 1821-1830, 2003.

[42] S. Furukawa, T. Fujita, M. Shimabukuro et al., "Increased oxidative stress in obesity and its impact on metabolic syndrome," Journal of Clinical Investigation, vol. 114, no. 12, pp. 1752-1761, 2004.

[43] M. Machado, P. Marques-Vidal, and H. Cortez-Pinto, "Hepatic histology in obese patients undergoing bariatric surgery," Journal of Hepatology, vol. 45, no. 4, pp. 600-606, 2006.

[44] R. S. Rector, J. P. Thyfault, Y. Wei, and J. A. Ibdah, "Nonalcoholic fatty liver disease and the metabolic syndrome: an update," World Journal of Gastroenterology, vol. 14, no. 2, pp. 185-192, 2008.

[45] M. Lazo and J. M. Clark, "The epidemiology of nonalcoholic fatty liver disease: a global perspective," Seminars in Liver Disease, vol. 28, no. 4, pp. 339-350, 2008.

[46] C. P. Day, "From fat to inflammation," Gastroenterology, vol. 130, no. 1, pp. 207-210, 2006.

[47] S.-M. Wang, L.-J. Lee, Y.-T. Huang, J.-J. Chen, and Y.-L. Chen, "Magnolol stimulates steroidogenesis in rat adrenal cells," British Journal of Pharmacology, vol. 131, no. 6, pp. 1172-1178, 2000.

[48] N. Crum-Cianflone, A. Dilay, G. Collins et al., "Nonalcoholic fatty liver disease among HIV-infected persons," Journal of Acquired Immune Deficiency Syndromes, vol. 50, no. 5, pp. 464473, 2009.
[49] M. C. Lewis, M. L. Phillips, J. P. Slavotinek, L. Kow, C. H. Thompson, and J. Toouli, "Change in liver size and fat content after treatment with Optifast very low calorie diet," Obesity Surgery, vol. 16, no. 6, pp. 697-701, 2006.

[50] J. H. Ho and C. Y. Hong, "Cardiovascular protection of magnolol: cell-type specificity and dose-related effects," Journal of Biomedical Science, vol. 19, article 70, 2012.

[51] J. Li, X. Shao, L. Wu et al., "Honokiol: an effective inhibitor of tumor necrosis factor- $\alpha$-induced up-regulation of inflammatory cytokine and chemokine production in human synovial fibroblasts," Acta Biochimica et Biophysica Sinica, vol. 43, no. 5, pp. 380-386, 2011. 


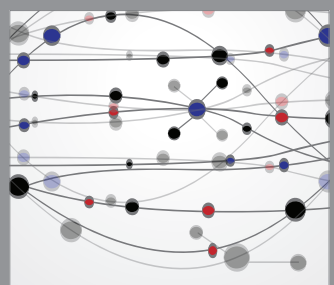

The Scientific World Journal
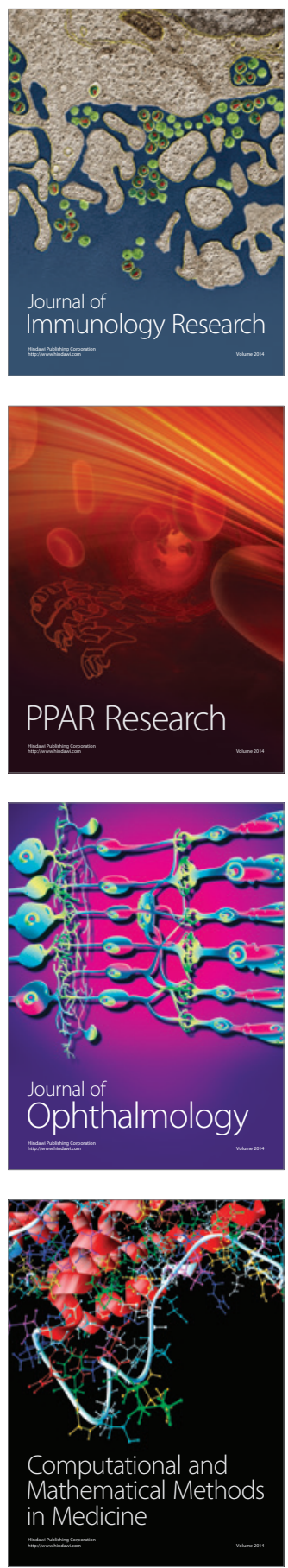

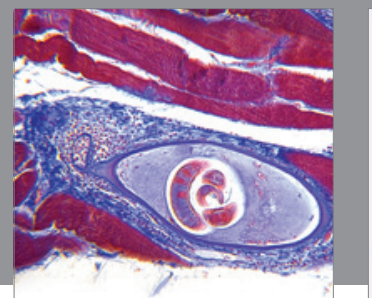

Gastroenterology

Research and Practice
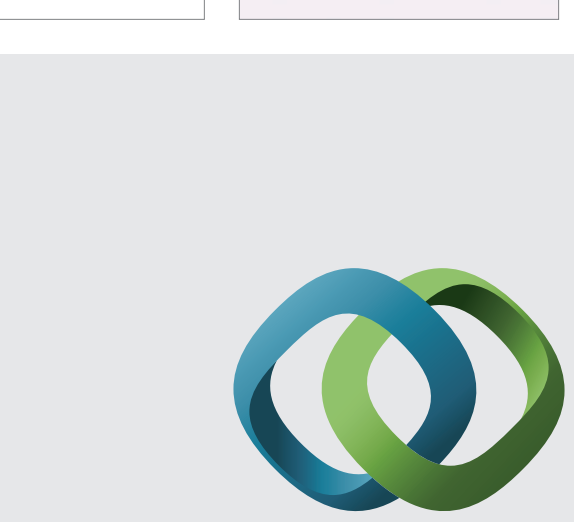

\section{Hindawi}

Submit your manuscripts at

http://www.hindawi.com
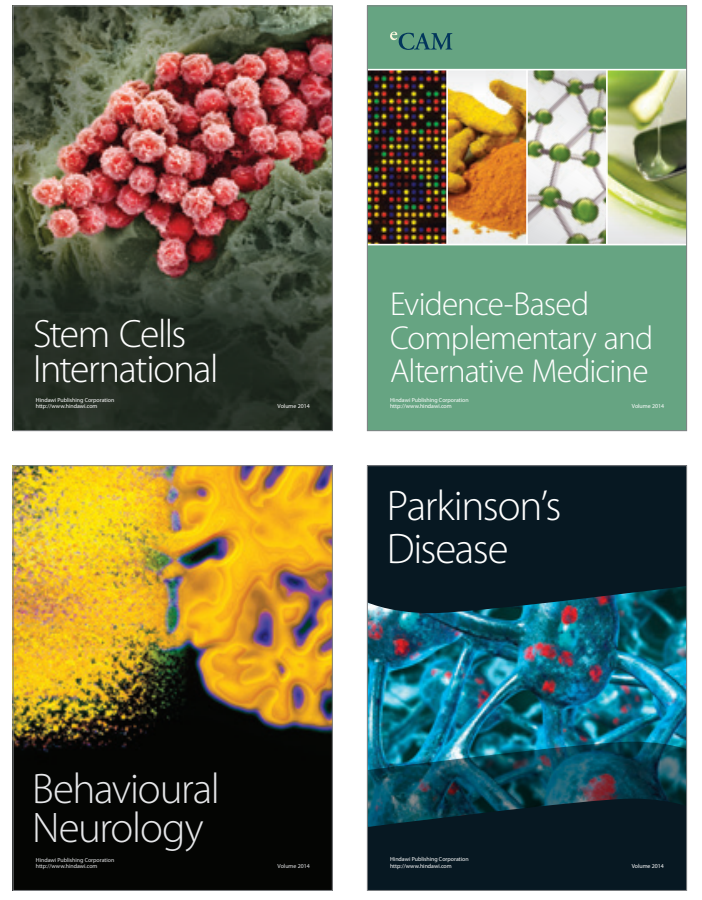
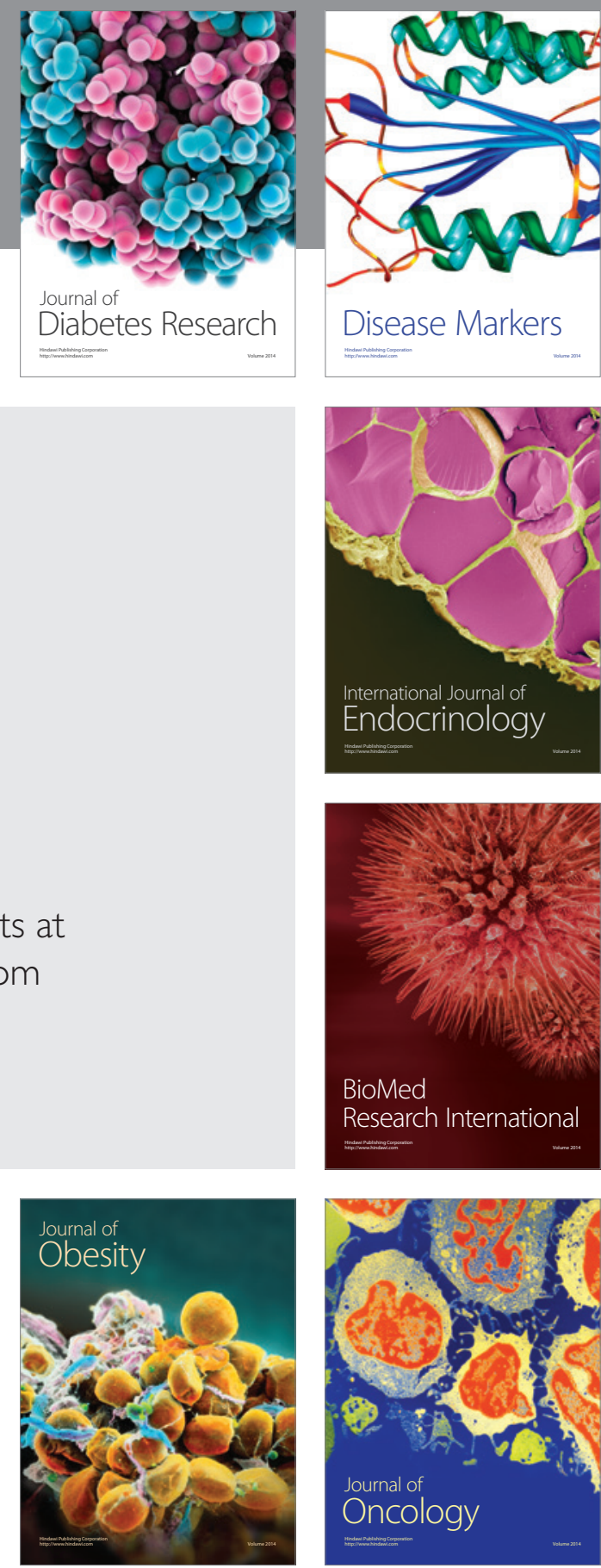

Disease Markers
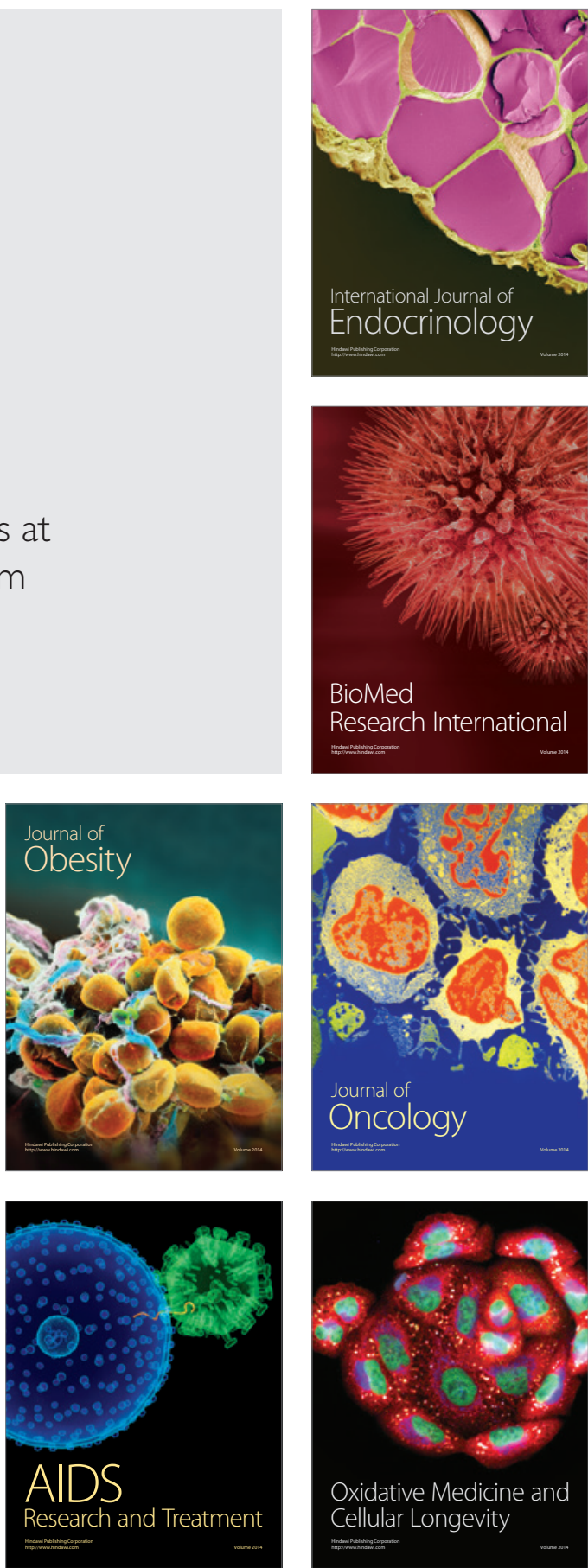